\title{
Reconstructing supersymmetric theories at high energy scales
}

\author{
G. A. Blair \\ Royal Holloway and Bedford New College, University of London, London, United Kingdom \\ and Deutsches Elektron-Synchrotron DESY, D-22603 Hamburg, Germany \\ W. Porod \\ Instituto de Física Corpuscular (IFIC), CSIC, E-46071-València, Spain \\ and Deutsches Elektron-Synchrotron DESY, D-22603 Hamburg, Germany \\ P. M. Zerwas \\ Deutsches Elektron-Synchrotron DESY, D-22603 Hamburg, Germany \\ and Institut für Theoretische Physik, Universität Wien, A-1090 Vienna, Austria
}

(Received 17 July 2000; published 12 December 2000)

\begin{abstract}
We have studied the reconstruction of supersymmetric theories at high scales by evolving the fundamental parameters from the electroweak scale upwards. Universal minimal supergravity and gauge mediated supersymmetry breaking have been taken as representative alternatives. Pseudo-fixed-point structures require the low-energy boundary values to be measured with high precision.
\end{abstract}

DOI: 10.1103/PhysRevD.63.017703

PACS number(s): 12.60.Jv, 11.10.Hi, 12.10.Kt

Supersymmetric theories in which fermionic and bosonic particles are assigned to common multiplets allow stable extrapolations to high energy scales of order $M_{U} \simeq 2 \times 10^{16}$ $\mathrm{GeV}$, where the electroweak and the strong couplings are expected to unify [1]. Since supersymmetry is not an exact symmetry, a variety of breaking mechanisms have been proposed, based on rather different physical ideas. Among these schemes are supergravity theories [2] and gauge mediated supersymmetry breaking [3]. The scales at which these mechanisms become effective extend from the grand unification scale near $10^{16} \mathrm{GeV}$ down to scales as low as order 1 $\mathrm{TeV}$. First indications about which of the scenarios could be realized in nature may be derived from the mass spectrum once supersymmetric particles are observed experimentally [4]. Moreover, dynamical signatures can be exploited for gauge mediated supersymmetry breaking, for example, such as delayed photon decays of the lightest neutralino or stau state [5].

In this Brief Report we address the extent to which the structure of supersymmetric theories at high scales can be reconstructed directly from future precision measurements of the properties of supersymmetric particles. Since the structure of the theory at the high scale cannot be assumed known a priori, top-down approaches may not reflect all facets of the theory in equal focus, while bottom-up approaches manifest the quality of the reconstruction in a more transparent form. The analysis is based on the assumption that no intermediate scale is realized between the electroweak scale and the fundamental high-energy scale. If present, however, the basic theory would be modified drastically and the evolution equations would have to be adjusted accordingly. While topdown approaches have been discussed frequently in the literature (see e.g. Refs. [5-8]), the direct reconstruction of the supersymmetric theory at the high scale, being much more difficult in practice, has not widely been addressed before. Theoretical elements in the context of fixed-point structures have been discussed in Refs. [9]. The analysis in this paper is phenomenological in nature, based on the experimental accuracies expected in the supersymmetric particle sector at the CERN Large Hadron Collider (LHC) and combined with expectations from future $e^{+} e^{-}$linear colliders (LC). The task relies on a comprehensive picture of the supersymmetric theory at the electroweak scale. Given the phenomenological complexity (see e.g. [10]), it will require the experimental information gathered in many years of collider operations.

As paradigm we will choose minimal supergravity (mSUGRA). The universal set of soft supersymmetry breaking parameters in this theory is generated near the Planck scale where supersymmetry breaking is mediated by gravity from a hidden sector [11]. Deviations from the universal values of the gaugino and scalar masses may be induced by the evolution down to the grand-unification scale (GUT) of the gauge couplings [12], or by contributions from non-singlet $F$ terms; the deviations may even be dramatic in superstring models (cf. Ref. [13] for details). Since the pattern of the mass terms may therefore not be regular at the GUT scale itself, the bottom-up approach is needed to uncover these more complicated structures.

We will confront the mSUGRA extrapolation with the alternative gauge mediated supersymmetry breaking (GMSB), characterized by a messenger scale $M_{m}$ in the range between $\sim 10 \mathrm{TeV}$ and $\sim 10^{6} \mathrm{TeV}$. In this scenario the mass parameters of particles carrying the same gauge quantum numbers squared are universal. The regularity for scalar masses would be observed at the scale $M_{m}$, while the gaugino mass parameters should unify at one-loop order at the GUT scale $M_{U}$ as before.

The extrapolation from the electroweak scale to the GUT scale in the mSUGRA scenario is based on the supersymmetric renormalization group equations [14]. To leading order, the gauge couplings and the gaugino and scalar mass parameters of soft supersymmetry breaking depend on the evolution coefficients 


$$
Z_{i}=\left[1+b_{i} \frac{\alpha_{U}}{4 \pi} \log \left(\frac{M_{U}}{M_{Z}}\right)^{2}\right]^{-1},
$$

with $b\left[S U_{3}, S U_{2}, U_{1}\right]=-3,1,33 / 5$; the scalar mass parameters also depend on the Yukawa couplings $h_{t}, h_{b}$, and $h_{\tau}$ of the top quark, bottom quark and $\tau$ lepton. Denoting the unified coupling at the GUT scale $M_{U}$ by $\alpha_{U}$, the universal gaugino mass by $M_{1 / 2}$, the universal sfermion and Higgs mass parameter by $M_{0}$, and the universal trilinear coupling by $A_{0}$, the renormalization group equations lead to the following relations for the low-scale parameters [15]:

$$
\begin{gathered}
\text { gauge couplings: } \alpha_{i}=Z_{i} \alpha_{U} \\
\text { gaugino mass parameters: } M_{i}=Z_{i} M_{1 / 2} \\
\text { scalar mass parameters: } M_{j}^{2}=M_{0}^{2}+c_{j} M_{1 / 2}^{2} \\
+\sum_{\beta=1,2} c_{j \beta}^{\prime} \Delta M_{\beta}^{2}
\end{gathered}
$$

$$
\text { trilinear couplings: } A_{k}=d_{k} A_{0}+d_{k}^{\prime} M_{1 / 2} \text {. }
$$

The coefficients $c_{j}\left[j=L_{l}, E_{l}, Q_{l}, U_{l}, D_{l}, H_{1,2} ; l=1,2,3\right]$ for the slepton and squark doublets/singlets of generation $l$, and for the Higgs doublets, are linear combinations of the evolution coefficients $Z_{i}$; the coefficients $c_{j \beta}^{\prime}$ are of order unity. The shifts $\Delta M_{\beta}^{2}$ are nearly zero for the first two families of sfermions, but they can be rather large for the third family and the Higgs mass parameters, depending on the coefficients $Z_{i}$, the universal parameters $M_{0}^{2}, M_{1 / 2}$ and $A_{0}$, and on the Yukawa couplings $h_{t}, h_{b}, h_{\tau}$. The coefficients $d_{k}$ of the trilinear couplings $A_{k}[k=t, b, \tau]$ depend on the corresponding Yukawa couplings and are approximately unity for the first two generations, while being $\mathrm{O}\left(10^{-1}\right)$ and smaller if the Yukawa couplings are large; the coefficients $d_{k}^{\prime}$, depending on gauge and Yukawa couplings, are of order unity.

In the present analysis the evolution equations have been solved to two-loop order [16] and threshold effects have been incorporated at the low scale [17]. We have checked that the points under study are compatible with $b \rightarrow s \gamma$ [18] and the $\rho$ parameter [19]. The mSUGRA point we have analyzed in detail is characterized by the following parameters: $M_{1 / 2}=190 \mathrm{GeV}, M_{0}=200 \mathrm{GeV}, A_{0}=550 \mathrm{GeV}, \tan \beta=30$, and $\operatorname{sgn}(\mu)=-$. The modulus of $\mu$ is calculated from the requirement of radiative electroweak symmetry breaking.

The initial experimental values, are generated by evolving the universal parameters down to the electroweak scale according to standard procedures $[20,17]$. These parameters define the experimental observables, including the supersymmetric particle masses and production cross sections, which are endowed with errors as in the continuum and from scanning the threshold regions; the threshold analysis provides in general the most accurate value. The analysis of the entire particle spectrum requires LC energies up to $1 \mathrm{TeV}$ and an integrated luminosity of about $1 \mathrm{ab}^{-1}$. The errors given in Ref. [8] are scaled in proportion to the masses of the spectrum. Moreover, they are inflated conservatively for particles
TABLE I. Representative experimental mass errors used in the fits to the mass spectra (see the text for details).

\begin{tabular}{lccc}
\hline \hline Particle & $\begin{array}{c}\text { M(GeV) } \\
\text { Mass }\end{array}$ & LHC & LHC+LC \\
\hline$h^{0}$ & 109 & 0.2 & 0.05 \\
$A^{0}$ & 191 & 3 & 1.5 \\
\hline$\chi_{1}^{+}$ & 133 & 3 & 0.11 \\
$\chi_{1}^{0}$ & 72.6 & 3 & 0.15 \\
\hline$\tilde{\nu}_{e}$ & 233 & 3 & 0.1 \\
$\tilde{e}_{1}$ & 217 & 3 & 0.15 \\
$\tilde{\nu}_{\tau}$ & 214 & 3 & 0.8 \\
$\tilde{\tau}_{1}$ & 154 & 3 & 0.7 \\
\hline$\tilde{u}_{1}$ & 466 & 10 & 3 \\
$\tilde{t}_{1}$ & 377 & 10 & 3 \\
\hline$\tilde{g}$ & 470 & 10 & 10 \\
\hline \hline
\end{tabular}

that decay predominantly to $\tau$ channels, according to typical reconstruction efficiencies such as those given in Ref. [21]. Typical examples are shown in Table I. The LC errors on the squark masses (see e.g. Ref. [22]) are set to an average value of $3 \mathrm{GeV}$; varying this error within a factor of 2 does not change the conclusions significantly since the measurement of the cross sections provides the maximal sensitivity in this sector. For the cross sections we use purely statistical errors, assuming a conservative reconstruction efficiency of $20 \%$. Parameter combinations from the fits to the spectrum and the cross sections which lead to charge and/or color breaking minima [23] are not accepted.

These observables are interpreted as the experimental input values for the evolution of the mass parameters in the bottom-up approach to the grand unification scale. The results for the evolution of the mass parameters to the GUT scale $M_{U}$ are shown in Fig. 1. The left-hand side (a) of the figure presents the evolution of the gaugino parameters $M_{i}$ which apparently is under excellent control, as is the extrapolation of the slepton mass parameter in Fig. 1(b). The accuracy deteriorates for both the squark mass parameters and the Higgs mass parameter $M_{\mathrm{H}_{2}}$. The origin of the differences between the errors for slepton, squark, and Higgs mass parameters can be traced back to the size of the coefficients in Eq. (4), for which typical examples read as follows:

$$
\begin{aligned}
M_{\widetilde{L}_{1}}^{2} \simeq M_{0}^{2}+0.52 M_{1 / 2}^{2} \\
M_{\widetilde{Q}_{1}}^{2} \simeq M_{0}^{2}+6.7 M_{1 / 2}^{2} \\
M_{\widetilde{H}_{2}}^{2} \simeq-0.18 M_{0}^{2}-2.2 M_{1 / 2}^{2}-0.35 A_{0} M_{1 / 2} \\
\quad-0.08 A_{0}^{2} .
\end{aligned}
$$

While the coefficients for sleptons are of order unity, the coefficient $c_{j}$ for squarks grows very large, $c_{j} \simeq 6.7$, so that small errors in $M_{1 / 2}^{2}$ are magnified by nearly an order of magnitude in the solution for $M_{0}$. By close inspection of Eq. (4) for the Higgs mass parameter, it turns out that the for- 

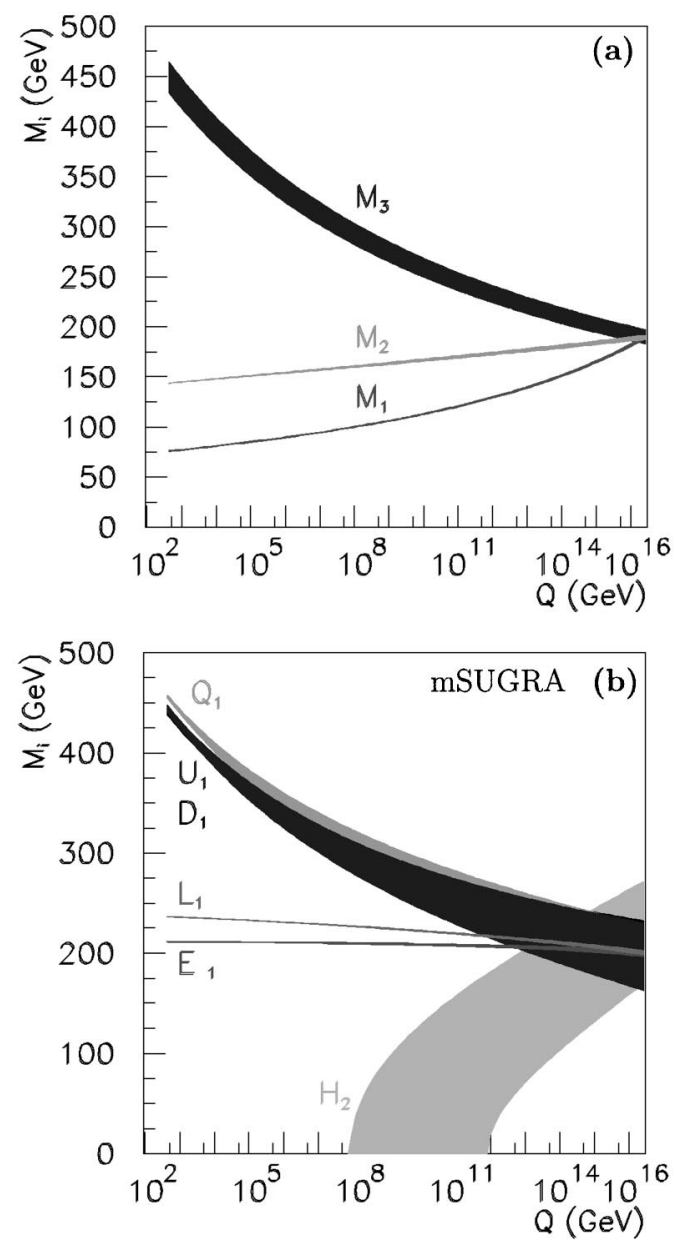

FIG. 1. mSUGRA: Evolution of (a) gaugino and (b) sfermion mass parameters in the bottom-up approach. The mSUGRA point probed is characterized by the parameters $M_{0}=200 \mathrm{GeV}, M_{1 / 2}$ $=190 \mathrm{GeV}, A_{0}=550 \mathrm{GeV}, \tan \beta=30$, and $\operatorname{sgn}(\mu)=(-)$. (The widths of the bands indicate the $95 \%$ C.L.)

mally leading $M_{0}^{2}$ part is nearly canceled by the $M_{0}^{2}$ part of $c_{j, \beta}^{\prime} \Delta M_{\beta}^{2}$. Inverting Eq. (4) for $M_{0}^{2}$ therefore gives rise to large errors in the Higgs case. A representative set of mass values and the associated errors, as evolving from the electroweak scale to $M_{U}$, is presented in Table II. The accuracy improves considerably if the LHC measurements are complemented by the high-precision LC measurements. Extracting the trilinear parameters $A_{k}$ is difficult and more refined analyses based on sfermion cross sections and Higgs and/or sfermion decays are necessary to determine these parameters more accurately. Moreover, the $A_{t}$ coupling, the best measured coupling among the $A_{k}$ parameters, shows a pseudo-fixed point behavior [9] since $d_{t} \simeq 0.2$ is small compared to $d_{t}^{\prime} \simeq 2$. All other trilinear couplings have only a weak impact on physical observables so that large experimental errors are expected. As a result, the fundamental parameter $A_{0}$ cannot be determined as precisely as the other parameters at the GUT scale.

It is apparent from this discussion that the errors in extracting the squark mass parameter $M_{0}$ depend strongly on whether $M_{0}$ is larger than $M_{1 / 2}$ (the case studied above), or
TABLE II. Representative mass parameters as determined at the electroweak scale and evolved to the GUT scale; based on LHC (left-hand side) and LC simulations (right-hand side). $L_{1,3}, Q_{1,3}$ are the slepton and squark isodoublet parameters of the first and third family. The minus sign (-) in front of $M_{H_{2}}$ refers to the negative value of $M_{\mathrm{H}_{2}}^{2}$ at the electroweak scale. (The errors quoted correspond to $1 \sigma$.)

\begin{tabular}{lcccc}
\hline \hline & \multicolumn{2}{c}{ LHC } & \multicolumn{2}{c}{ LC } \\
& exp. input & GUT value & exp. input & GUT value \\
\hline$M_{1}$ & $75.6 \pm 3.2$ & $189.6 \pm 7.6$ & $75.6 \pm 0.2$ & $189.6 \pm 0.7$ \\
$M_{2}$ & $143.6 \pm 3.1$ & $190.6 \pm 3.8$ & $143.6 \pm 0.2$ & $189.4 \pm 0.9$ \\
$M_{3}$ & $452.3 \pm 11.9$ & $190.1 \pm 5.7$ & $452.3 \pm 9$ & $190.0 \pm 4.2$ \\
\hline$M_{L_{1}}$ & $236.8 \pm 2.1$ & $200.6 \pm 6.9$ & $236.8 \pm 0.1$ & $200.5 \pm 0.9$ \\
$M_{Q_{1}}$ & $459.6 \pm 7.4$ & $200.7 \pm 30.5$ & $459.7 \pm 0.6$ & $200 \pm 18$ \\
\hline$M_{L_{3}}$ & $218.6 \pm 2.8$ & $199.5 \pm 12.3$ & $218.6 \pm 0.6$ & $196.5 \pm 7.2$ \\
$M_{Q_{3}}$ & $392 \pm 45$ & $192 \pm 251$ & $391.2 \pm 1.0$ & $233 \pm 46$ \\
\hline$M_{H_{1}}$ & $132.4 \pm 12$ & $361 \pm 324$ & $132.4 \pm 1.5$ & $224 \pm 90$ \\
$\left|M_{H_{2}}\right|$ & $(-) 251.9 \pm 2.2$ & $279 \pm 98$ & $(-) 251.9 \pm 0.2$ & $211 \pm 27$ \\
\hline$A_{\tau}$ & $101 \pm 2590$ & $210 \pm 432$ & $100 \pm 92$ & $319 \pm 340$ \\
$A_{b}$ & $-125 \pm 3920$ & $806 \pm 1292$ & $-126 \pm 286$ & $129 \pm 571$ \\
$A_{t}$ & $-186 \pm 39$ & $608 \pm 169$ & $-186.3 \pm 3.2$ & $505 \pm 81$ \\
\hline \hline
\end{tabular}

whether $M_{0}$ is smaller than $M_{1 / 2}$. As an example in the latter case, the large Yukawa couplings of the third generation can enhance the pseudo-fixed point behavior, leading to large errors for $M_{0}$ in the third generation.

Inspecting Fig. 1(b) leads to the conclusion that the topdown approach eventually may generate an incomplete picture. Global fits based on mSUGRA without allowing for deviations from universality are dominated by $M_{1,2}$ and the slepton mass parameters due to the pseudo-fixed point behavior of the squark mass parameters. Therefore, the structure of the theory in the squark sector is not scrutinized stringently at the unification scale in the top-down approach. By contrast, the bottom-up approach very clearly demonstrates

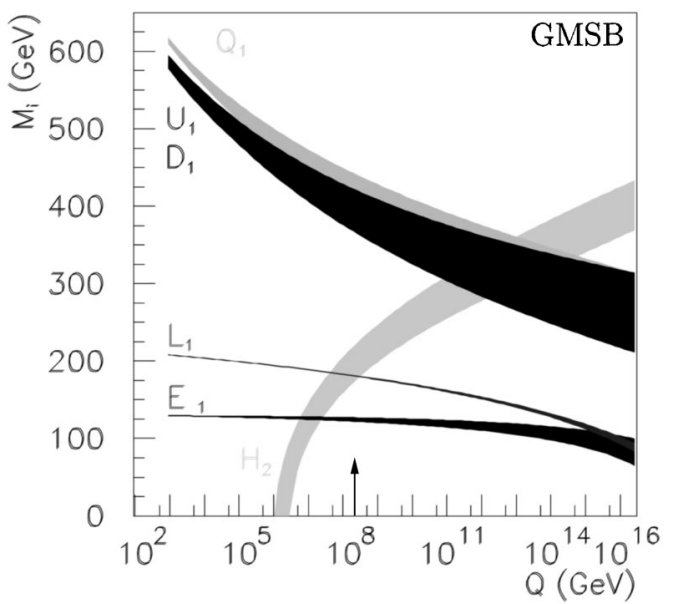

FIG. 2. GMSB: Evolution of sfermion mass parameters in the bottom-up approach. The GMSB point has been chosen as $M_{m}$ $=2 \times 10^{5} \mathrm{TeV}, \Lambda=28 \mathrm{TeV}, N_{5}=3, \tan \beta=30$, and $\operatorname{sgn}(\mu)=$ $(-)$. (The widths of the bands indicate the $95 \%$ C.L.) 
the extent to which the theory can be tested at the high scale.

To confront the mSUGRA analysis with an alternative scenario, the analysis has been repeated at energies up to 1.5 $\mathrm{TeV}$ for gauge mediated supersymmetry breaking (GMSB). Regularity among particles carrying the same gauge quantum numbers squared should in this scenario be observed in the evolution of mass parameters at the messenger scale. The evolution of the sfermion mass parameters of the first/second generation and the Higgs mass parameter $M_{H_{2}}$ is presented in Fig. 2. It is obvious that $M_{H_{2}}$ approaches the mass parameter for the left-chiral sleptons at the GMSB scale. Moreover, the figure clearly demonstrates that GMSB will not be confused with the mSUGRA scenario since no more regularity can be observed at the GUT scale $M_{U}$.

In summary, the model-independent reconstruction of the fundamental supersymmetric theory at the high scale, the grand unification scale $M_{U}$ in supergravity or the intermediate scale $M_{m}$ in gauge mediated supersymmetry breaking, appears feasible. Regular patterns can be observed by evolv- ing the gaugino and scalar mass parameters from the measured values at the electroweak scale to the high scales. The accuracy is significantly improved if, in addition to the LHC input values, high-precision LC values are also included. The future experimental input from LC is particularly important if the universality at the GUT scale is (slightly) broken. Precision data are therefore essential for stable extrapolations to high energy scales.

\section{ACKNOWLEDGMENTS}

Thanks for discussions go to M. Peskin and D. Pierce. W.P. and G.B. are grateful to DESY for the warm hospitality during their stay in Hamburg, and P.M.Z. is grateful to the Institut für Theoretische Physik of Vienna University. W.P. is supported by the Spanish "Ministerio de Educacion y Cultura" under the contract SB97-BU0475382, by Spanish DGICYT grants PB98-0693, and by the EEC under the TMR contract ERBFMRX-CT96-0090.
[1] S. Dimopoulos, S. Raby, and F. Wilczek, Phys. Rev. D 24, 1681 (1981); L. E. Ibanez and G. G. Ross, Phys. Lett. 105B, 439 (1981); U. Amaldi, W. de Boer, and H. Fürstenau, Phys. Lett. B 260, 447 (1991); P. Langacker and M. Luo, Phys. Rev. D 44, 817 (1991); J. Ellis, S. Kelley, and D. V. Nanopoulos, Phys. Lett. B 260, 161 (1991).

[2] A. H. Chamseddine, R. Arnowitt, and P. Nath, Phys. Rev. Lett. 49, 970 (1982).

[3] M. Dine and A. E. Nelson, Phys. Rev. D 48, 1277 (1993).

[4] J. Bagger, K. Matchev, D. Pierce, and R. Zhang, Phys. Rev. D 55, 3188 (1997); M. E. Peskin, hep-ph/0002041.

[5] S. Ambrosanio and G. A. Blair, Eur. Phys. J. C 12, 287 (2000).

[6] H. Murayama and M. E. Peskin, Annu. Rev. Nucl. Part. Sci. 46, 533 (1996); ECFA/DESY LC Working Group, E. Accomando et al., Phys. Rep. 299, 1 (1998); P. M. Zerwas, hep-ph/0003221.

[7] I. Hinchliffe et al., Phys. Rev. D 55, 5520 (1997); Atlas Collaboration, Technical Design Report 1999, Vol. II, CERN/ LHC/99-15, ATLAS TDR 15.

[8] G. A. Blair and U. Martyn, hep-ph/9910416.

[9] M. Carena et al., Nucl. Phys. B491, 103 (1997); S. A. Abel and B. Allanach, Phys. Lett. B 415, 371 (1997).

[10] S. Y. Choi et al., Eur. Phys. J. C 14, 535 (2000); M. Brhlik and G. L. Kane, Phys. Lett. B 437, 331 (1998); J. L. Feng and T. Moroi, Phys. Rev. D 56, 5962 (1997).
[11] H. P. Nilles, Phys. Rep. 110, 1 (1984).

[12] N. Polonsky and A. Pomarol, Phys. Rev. Lett. 73, 2292 (1994).

[13] C. H. Chen, M. Drees, and J. F. Gunion, Phys. Rev. D 55, 330 (1997); 60, 039901(E) (1999).

[14] K. Inoue, A. Kakuto, H. Komatsu, and S. Takeshita, Prog. Theor. Phys. 68, 927 (1982); 70, 330(E) (1983).

[15] C. Kounnas, I. Pavel, G. Ridolfi, and F. Zwirner, Phys. Lett. B 354, 322 (1995).

[16] S. Martin and M. Vaughn, Phys. Rev. D 50, 2282 (1994); Y. Yamada, ibid. 50, 3537 (1994); I. Jack and D. R. T. Jones, Phys. Lett. B 333, 372 (1994).

[17] J. Bagger, K. Matchev, D. Pierce, and R. Zhang, Nucl. Phys. B491, 3 (1997).

[18] A. L. Kagan and M. Neubert, Eur. Phys. J. C 7, 5 (1999); D. A. Demir, A. Masiero, and O. Vives, Phys. Rev. D 61, 075009 (2000).

[19] M. Drees and K. Hagiwara, Phys. Rev. D 42, 1709 (1990).

[20] H. Arason et al., Phys. Rev. D 46, 3945 (1992).

[21] M. M. Nojiri, K. Fujii, and T. Tsukamoto, Phys. Rev. D 54, 6756 (1996).

[22] J. L. Feng and D. E. Finnell, Phys. Rev. D 49, 2369 (1994); A. Bartl, H. Eberl, S. Kraml, W. Majerotto, and W. Porod, hep-ph/0002115.

[23] J.A. Casas, A. Lleyda, and C. Munoz, Nucl. Phys. B471, 3 (1996). 